\title{
A systematic review of the efficacy and safety of a purified, pasteurized C1 inhibitor concentrate for the treatment of patients with type I or II hereditary angioedema
}

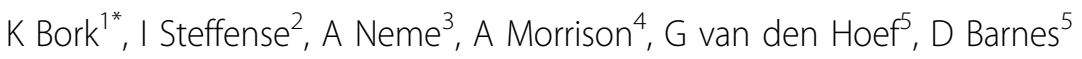 \\ From Canadian Society of Allergy and Clinical Immunology Annual Scientific Meeting 2009 \\ Halifax, Canada. 22-25 October 2009
}

\section{Background}

Hereditary angioedema (HAE) is a rare disorder characterized by a congenital deficiency of the C1-inhibitor. It is a potentially fatal illness as laryngeal edema may lead to asphyxiation. Treatment of HAE is mainly performed with C1-inhibitor concentrate, icatibant, attenuated androgens, and antifibrinolytic agents. We conducted a systematic review to evaluate the efficacy and safety of a purified, pasteurized C1-inhibitor concentrate in the treatment of patients with type I or II HAE.

\section{Method}

An electronic search of five databases (Medline, EMBASE, Biosis Previews, CINAHL and the Cochrane Library) was performed with no language or date restrictions applied. Two reviewers used pre-defined criteria for inclusion of studies and the data were tabulated and analyzed. The data were found to be inappropriate for meta-analysis, and thus a qualitative synthesis was performed.

\section{Results}

We found a total of 63 studies that met our inclusion criteria. Treatment with the C1-inhibitor concentrate was consistently associated with clinically significant reductions in both the time to onset of symptom relief and duration of acute attacks. There were no cases of angioedema in patients who received the C1-inhibitor concentrate as short-term prophylaxis, and use of the C1-inhibitor concentrate as a long-term prophylactic agent resulted in a significant decrease in the frequency of attacks. No serious AEs were reported in any patients who were treated with the $\mathrm{C} 1$-inhibitor concentrate, and there were no treatment-related AEs as far as the injections were performed correctly. Treatment with the C1inhibitor concentrate was not associated with transmission of viruses or development of autoantibodies.

\section{Conclusion}

Results of this systematic review found the C1-inhibitor concentrate to be an effective option in the treatment of acute HAE attacks, and as a short- and long-term prophylactic agent.

\section{Author details}

${ }^{1}$ University of Mainz Mainz, Germany. ${ }^{2}$ Meta-Business Consulting Inc.

Ottawa, Canada. ${ }^{3}$ Clinsig Research Consulting Inc., Brampton, Canada. ${ }^{4}$ MetaBusiness Consulting Inc., Ottawa, Canada. ${ }^{5}$ CSL Behring Canada, Inc., Ottawa, Canada.

Published: 12 May 2010 